\title{
Dietary palmitate and oleate differently modulate insulin sensitivity in human skeletal muscle
}

\author{
Theresia Sarabhai ${ }^{1,2,3} \cdot$ Chrysi Koliaki $^{2,3} \cdot$ Lucia Mastrototaro ${ }^{2,3} \cdot$ Sabine Kahl ${ }^{2,3} \cdot$ Dominik Pesta $^{2,3}$. \\ Maria Apostolopoulou ${ }^{1,2,3} \cdot$ Martin Wolkersdorfer $^{4}$ - Anna C. Bönner ${ }^{2,3} \cdot$ Pavel Bobrov $^{3,5} \cdot$ Daniel F. Markgraf ${ }^{2,3}$. \\ Christian Herder ${ }^{1,2,3}$. Michael Roden ${ }^{1,2,3}$
}

Received: 17 May 2021 / Accepted: 16 August 2021 / Published online: 26 October 2021

(C) The Author(s) 2021

\begin{abstract}
Aims/hypothesis Energy-dense nutrition generally induces insulin resistance, but dietary composition may differently affect glucose metabolism. This study investigated initial effects of monounsaturated vs saturated lipid meals on basal and insulinstimulated myocellular glucose metabolism and insulin signalling.

Methods In a randomised crossover study, 16 lean metabolically healthy volunteers received single meals containing safflower oil (SAF), palm oil (PAL) or vehicle (VCL). Whole-body glucose metabolism was assessed from glucose disposal $\left(R_{\mathrm{d}}\right)$ before and during hyperinsulinaemic-euglycaemic clamps with $\mathrm{D}-\left[6,6-{ }^{2} \mathrm{H}_{2}\right]$ glucose. In serial skeletal muscle biopsies, subcellular lipid metabolites and insulin signalling were measured before and after meals.

Results SAF and PAL raised plasma oleate, but only PAL significantly increased plasma palmitate concentrations. SAF and PAL increased myocellular diacylglycerol and activated protein kinase $\mathrm{C}$ (PKC) isoform $\theta(p<0.05)$ but only PAL activated PKC $\varepsilon$. Moreover, PAL led to increased myocellular ceramides along with stimulated $\mathrm{PKC} \zeta$ translocation $(p<0.05$ vs SAF). During clamp, SAF and PAL both decreased insulin-stimulated $R_{\mathrm{d}}(p<0.05$ vs VCL), but non-oxidative glucose disposal was lower after PAL compared with SAF $(p<0.05)$. Muscle serine ${ }^{1101}$-phosphorylation of IRS-1 was increased upon SAF and PAL consumption $(p<0.05)$, whereas PAL decreased serine ${ }^{473}$-phosphorylation of Akt more than SAF $(p<0.05)$.

Conclusions/interpretation Lipid-induced myocellular insulin resistance is likely more pronounced with palmitate than with oleate and is associated with PKC isoforms activation and inhibitory insulin signalling.

Trial registration ClinicalTrials.gov.NCT01736202.

Funding German Federal Ministry of Health, Ministry of Culture and Science of the State North Rhine-Westphalia, German Federal Ministry of Education and Research, European Regional Development Fund, German Research Foundation, German Center for Diabetes Research.
\end{abstract}

Michael Roden

michael.roden@ddz.de

1 Department of Endocrinology and Diabetology, Medical Faculty and University Hospital Düsseldorf, Heinrich-Heine-University, Düsseldorf, Germany

2 Institute for Clinical Diabetology, German Diabetes Center, Leibniz Institute for Diabetes Research at Heinrich-Heine-University, Düsseldorf, Germany

3 German Center for Diabetes Research, Partner Düsseldorf, Neuherberg, Germany

Keywords Glucose metabolism · Insulin signalling · Lipotoxicity - Monounsaturated fatty acids - Saturated fat . Saturated fat $\cdot$ Skeletal muscle

4 Landesapotheke Salzburg, Department of Production, Hospital Pharmacy, Salzburg, Austria

$\begin{array}{ll}\text { Abbreviations } \\ \text { ALT } & \text { Alanine aminotransferase } \\ \text { AST } & \text { Aspartate aminotransferase } \\ \text { aPKC } & \text { Atypical protein kinase C } \\ \text { BSA } & \text { Body surface area } \\ \text { BW } & \text { Body weight } \\ \text { CSA } & \text { Citrate synthase activity } \\ \text { DAG } & \text { Diacylglycerol } \\ \text { DDZ } & \text { German Diabetes Center } \\ \text { EGP } & \text { Endogenous glucose production } \\ \text { FA } & \text { Fatty acid } \\ \text { FAME } & \text { FA methyl ester }\end{array}$

Leibniz Cor Biometrics and Epidemiology, German Diabetes Center, Düsseldorf, Germany 


\section{Research in context}

\section{What is already known about this subject?}

- The western diet, high in saturated fat, is associated with insulin resistance (obesity/type 2 diabetes), and current guidelines recommend a diet high in monounsaturated fat

- Previous studies have shown that skeletal muscle insulin resistance in obesity and type 2 diabetes is associated with activation of the diacylglycerol (DAG)-novel protein kinase $C \theta(n P K C \theta)$ pathway, which has also been shown to be effective in glucose-tolerant individuals after i.v. infusion of monounsaturated fatty acids (FAs)

\section{What is the key question?}

- Does the degree of different FA saturation (monounsaturated vs saturated) have a differential effect on muscle insulin signalling in healthy individuals?

\section{What are the new findings?}

- This study shows that a single lipid load, independent of its FAs, induces whole-body and hepatic insulin resistance associated with the myocellular DAG-nPKC $\theta-$ serine ${ }^{1101}-$ IRS pathway

- Only saturated fat intake was associated with increased levels of myocellular ceramide, which may contribute to insulin resistance in skeletal muscle via activation of atypical $\mathrm{PKC}$ and protein phosphatase $2 \mathrm{~A}$, which inhibit serine ${ }^{473}$-phopshorylation of Akt

\section{How might this impact on clinical practice in the foreseeable future?}

- This study contributes to a more comprehensive understanding of lipid-induced myocellular insulin resistance and the effects of different degrees of FA saturation on insulin signalling, underlining the relevance of lipotoxic pathways as therapeutic targets to prevent and reverse muscle insulin resistance in humans

$\begin{array}{ll}\text { GIP } & \text { Gastric inhibitory polypeptide } \\ \text { GLP-1 } & \text { Glucagon-like peptide-1 } \\ \text { JNK } & \text { Jun N-terminal kinase } \\ \text { GOX } & \text { Glucose oxidation } \\ \text { iAUC } & \text { Incremental AUC } \\ \text { LOX } & \text { Lipid oxidation } \\ \text { NOXGD } & \text { Non-oxidative glucose disposal } \\ \text { nPKC } & \text { Novel protein kinase C } \\ \text { PAL } & \text { Palm oil } \\ \text { PP2A } & \text { Protein phosphatase 2A } \\ R_{\mathrm{d}} & \text { Rate of glucose disposal } \\ \text { REE } & \text { Resting energy expenditure } \\ \text { SAF } & \text { Safflower oil } \\ \text { VCL } & \text { Vehicle }\end{array}$

\section{Introduction}

Western-style diets, as defined by high intake of energy and fat, have been related to the rising prevalence of insulinresistant states such as obesity and type 2 diabetes $[1,2]$. Although current guidelines recommend diets low in saturated fatty acid (FA) for type 2 diabetes and CVD, the evidence remains limited $[3,4]$.

Previously, we have demonstrated that the skeletal muscle insulin resistance in obesity and type 2 diabetes associates with activation of the myocellular diacylglycerol (DAG)novel protein kinase $\mathrm{C}$ (nPKC) isoform $\theta$ pathway, an inhibitory cascade of proximal insulin signalling $[5,6]$. In line with results from preclinical models [7], we showed that this pathway is also operative in glucose-tolerant humans upon i.v. infusion of lipid emulsions containing predominately monounsaturated FA, as well as in obese individuals and those with type 2 diabetes without any infusion [6]. Of note, this study did not detect changes in other mechanisms that have been postulated to induce insulin resistance, such as sphingolipid mediators, abnormal mitochondrial function or low-grade inflammation, in some [8] but not all previous human studies $[6,9,10]$. Among other causes, different outcomes may result from the degree of NEFA saturation, which can differently affect metabolism and risk of type 2 diabetes and CVD, as saturated FA, but not monounsaturated FA, are considered harmful [11, 12]. Monounsaturated FA availability is known to activate the DAG-nPKC $\theta$ pathway [6], whereas saturated FA can increase intracellular ceramides and stimulate protein phosphatase $2 \mathrm{~A}(\mathrm{PP} 2 \mathrm{~A})$ and the atypical protein kinase $\mathrm{C}$ 
(aPKC) isoform $\zeta$, which inhibit activation of Akt [1]. On the other hand, oleate may even protect against palmitate-induced insulin resistance, as demonstrated in L6 myotubes [13]. However, the temporal sequence of the molecular events in human skeletal muscle upon ingestion of differently composed lipid meals remains unknown.

We aimed to compare the acute effects of saturated fat (palm oil [PAL]) and monounsaturated fat (safflower oil [SAF]) with water (vehicle [VCL]) on the following variables in young, lean and metabolically healthy humans (ESM Table 1): (1) whole-body insulin sensitivity; (2) (sub)cellular distribution of lipid intermediates; (3) insulin signalling; and (4) mitochondrial oxidative capacity in skeletal muscle. Thus, we performed comprehensive metabolic phenotyping using serial biopsies before and after $2 \mathrm{~h}, 4 \mathrm{~h}$, as well as $7 \mathrm{~h}$ of the respective interventions (Fig. 1 and ESM Fig. 1).

\section{Methods}

Volunteers The study was registered at Clinicaltrials.gov (registration no. NCT01736202), approved by the ethics board of Heinrich Heine University of Düsseldorf (reference 3107) and performed according to the Declaration of Helsinki, 2013. All participants (ten men, six women) gave their written informed consent prior to enrolment in this randomised, placebo-controlled, crossover trial. Inclusion criteria were age of 20-40 years and BMI of $20-25 \mathrm{~kg} / \mathrm{m}^{2}$. Exclusion criteria were family history of diabetes, dysglycaemia, menstrual irregularities, history of smoking, alcohol or drug abuse and other acute or chronic diseases including cancer, as well as any medication intake affecting insulin sensitivity, immune system or lipid metabolism. All volunteers had a screening visit for medical history and clinical examination, lean body mass assessment, routine laboratory tests and a $75 \mathrm{~g}$ OGTT. Eligible participants were instructed to maintain and record a carbohydrate-rich diet and to avoid intense physical activity for 3 days prior to all study days. Female participants were examined only between days 5 and 9 of their menstrual cycle. All participants were randomly assigned to the three study days, spaced by an interval of 3 weeks (Fig. 1).

Randomisation The random allocation sequence (1:1) was generated by an expert statistician (PB) using SAS software, version 9.3. (SAS Institute, Cary, NC, USA). Participants were randomly assigned to their treatment order by an independent person not involved in the study at the German Diabetes Center (DDZ). The randomisation list was kept by this person and was not accessible to the study personnel. Study participants, medical staff and researchers were blinded until completion of the study.

Experimental protocol The study day comprised three periods (Fig. 1): pre-basal ( $-120 \mathrm{~min}$ to $0 \mathrm{~min})$; basal $(0 \mathrm{~min}$ to +360 min); and clamp (+360 min to $+480 \mathrm{~min}$ ). All participants arrived at DDZ, after a $10 \mathrm{~h}$ overnight fast at $07: 30 \mathrm{~h}$ (-120 min; Fig. 1). Two i.v. catheters were inserted into contralateral forearm veins. A continuous infusion $\left.(0.036 \mathrm{mg} \text { [kg body weight }(\mathrm{BW})]^{-1} \mathrm{~min}^{-1}\right)$ of D-[6,6- ${ }^{2} \mathrm{H}_{2}$ ]glucose (99\% enrichment; Cambridge Isotope Laboratories, Andover, MA, USA) was given from $-120 \mathrm{~min}$ to $+480 \mathrm{~min}$ [11], following a 10 -min priming bolus (0.36 mg $[\mathrm{kg} \mathrm{BW}]^{-1} \mathrm{~min}^{-1}[\mathrm{mg} / \mathrm{dl}$ fasting blood glucose $\left.]\right)$. At 0 min, participants received one of three interventions: (1) PAL $\sim 1.18 \mathrm{~g} / \mathrm{kg} \mathrm{BW}$ (48\% saturated FA, 35\% monounsaturated FA, $15 \%$ polyunsaturated FA; Biopalm, Landkrone, Hamburg, Germany); (2) SAF $~ 1.18$ g/kg BW (8\% saturated FA, $65 \%$ monounsaturated FA, $23 \%$ polyunsaturated FA;

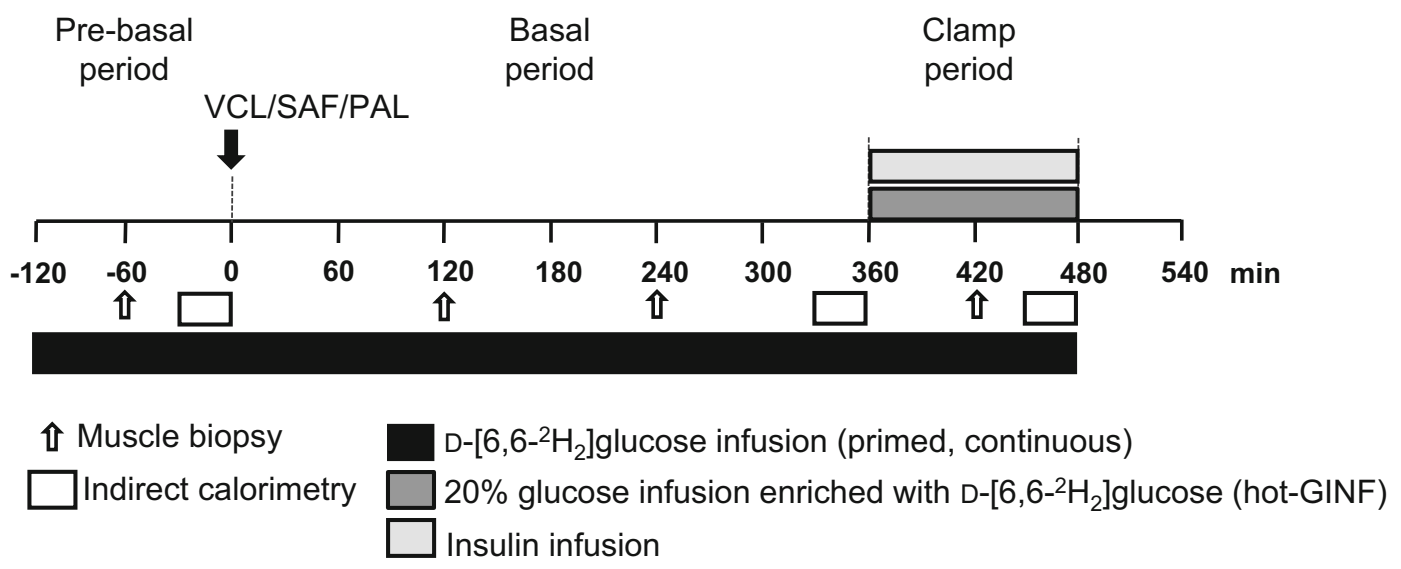

Fig. 1 Study design. Lean, healthy adults (10 male and 6 female) randomly ingested either one dose of PAL, SAF or VCL (water) at time point $0 \mathrm{~min}$ on three occasions during a period of 12 weeks. Starting at $-120 \mathrm{~min}, \mathrm{D}-\left[6,6-{ }^{2} \mathrm{H}_{2}\right]$ glucose was infused up to $+480 \mathrm{~min}$. Muscle

biopsies were taken at time points $-60 \mathrm{~min},+120 \mathrm{~min},+240 \mathrm{~min}$ and +420 min. From +360 min to $+480 \mathrm{~min}$, a hyperinsulinaemiceuglycaemic clamp test was performed according to the 'hot' glucose infusion (hot-GINF) protocol 
Mazola, Elmshorn, Germany); or (3) control (VCL) $1.18 \mathrm{ml} /$ kg BW of bottled still water (Ahrtal Quelle, Sinzig, Germany). Participants drank $92 \mathrm{~g}$ (if BW $>70 \mathrm{~kg}$ ) or $80 \mathrm{~g}$ (if BW $<70 \mathrm{~kg}$ ) of the lipid drinks (heated up to $60^{\circ} \mathrm{C}$, mixed with $1.84 \mathrm{~g}$ or $1.60 \mathrm{~g}$ emulsifier [Glice; Texturas, Albert y Ferran Adria, Barcelona, Spain], 9 g or 8 g sugar-free vanilla syrup [Torani, San Francisco, CA, USA] and $81.2 \mathrm{ml}$ or $70.4 \mathrm{ml}$ bottled still water, respectively. The VCL drink contained $173.2 \mathrm{ml}$ or $150.4 \mathrm{ml}$ still water. Participant and investigator were blinded to treatment sequence. Each intervention ( $0 \mathrm{~min})$ was followed by a hyperinsulinaemic-euglycaemic clamp: a bolus of $80 \mathrm{mU} \mathrm{m}^{-2}$ (body surface area [BSA]) $\mathrm{min}^{-1}$ for $8 \mathrm{~min}$, followed by continuous infusion of $40 \mathrm{mU} \mathrm{m}^{-2}$ (BSA) $\min ^{-1}$ of human short-acting insulin (Insuman Rapid; Sanofi, Frankfurt, Germany) from $+360 \mathrm{~min}$ to $+480 \mathrm{~min}$. Blood glucose was maintained at $5 \mathrm{mmol} / \mathrm{l}$ by adapting rates of the $20 \%$ glucose infusion (Braun, Melsungen, Germany), enriched with D-[6,6- ${ }^{2} \mathrm{H}_{2}$ ]glucose (ESM Fig. 2). Further blood samples were collected at timed intervals [6]. Vital function (heart rate, BP, body temperature) was monitored every $60 \mathrm{~min}$.

Indirect calorimetry Indirect calorimetry was performed in the canopy mode using Vmax Encore 29n (CareFusion, Höchberg, Germany) during the last $30 \mathrm{~min}$ of the pre-basal, basal and clamp periods $[5,11]$.

Skeletal muscle biopsy Biopsies were taken at $-60,+120$, +240 and +420 min from the vastus lateralis muscle of both legs. Under local anaesthesia, muscle specimens were obtained by a modified Bergström needle with suction and were immediately blotted free of extramyocellular tissue or blood, frozen in liquid nitrogen, weighed and stored at $-80^{\circ} \mathrm{C}[5]$.

Plasma FA analysis NEFA were analysed as their FA methyl esters (FAMEs) using GC-MS, as described in detail elsewhere $[10,14]$. Direct transesterification of all classes of lipids was carried out in a one-step reaction [14]. In brief, lipids were extracted from plasma after addition of internal standard (heptadecanoic acid) using isopropyl alcohol-heptane-sulfuric acid (40:10:1) (Merck, Darmstadt, Germany) and 0.01\% butylated hydroxytoluene ( $\geq 99 \%$, B1378; Sigma-Aldrich, St Louis, MO, USA). Lipids were separated by thin-layer chromatography using heptane-diethylether-acetic acid (80:30:1) (Merck) as mobile phase. FAs were extracted from silica gel in benzol-methanol (1:4) (Merck) overnight and derivatised to their corresponding methyl esters by addition of acetyl chloride (Merck) and incubation at $100^{\circ} \mathrm{C}$ for $1 \mathrm{~h}$. After addition of benzol (Merck) and centrifugation, the FAMEcontaining supernatant fraction was analysed on a Hewlett Packard 6890 gas chromatograph (Palo Alto, CA, USA) interfaced to a Hewlett Packard 5975 mass selective detector.
Calibration curves of reference FAs were processed in parallel for quantification [10].

Myocellular lipids For quantification of lipid metabolites in subcellular compartments, lipids were extracted, purified and analysed from frozen tissue samples, using liquid chromatography tandem-mass spectrometry (LC-MS/MS) as previously described $[5,15]$.

Myocellular signalling Insulin signalling was assessed by western blotting. Total soluble proteins were extracted from approximately $30 \mathrm{mg}$ of frozen skeletal muscle and homogenised in $300 \mu \mathrm{l}$ of lysis buffer $(25 \mathrm{~mol} / \mathrm{l}$ Tris- $\mathrm{HCl}, 1 \mathrm{mmol} / \mathrm{l}$ EDTA, $150 \mathrm{mmol} / 1 \mathrm{NaCl}, 0.20 \% \mathrm{NP}-40)$ with protease (cOmplete Tablets, EASYpack; Roche Diagnostics, Basel, Switzerland) and phosphatase (PhosSTOP, EASYpack; Roche Diagnostics) inhibitors. Activities of PKCs were assessed from the ratios of the protein contents in membrane and cytosol fractions upon differential centrifugation. A total of $50 \mathrm{mg}$ of frozen muscle tissue was homogenised in $300 \mu \mathrm{l}$ of lysis buffer A $(25 \mathrm{mmol} / 1$ Tris-HCL, $1 \mathrm{mmol} / 1$ EDTA, $150 \mathrm{mmol} / 1 \mathrm{NaCl}, 0.20 \% \mathrm{NP}-40$, with protease and phosphatase inhibitors), centrifuged $\left(100,000 \mathrm{~g}, 1 \mathrm{~h}\right.$ at $\left.4^{\circ} \mathrm{C}\right)$, and the supernatant fraction containing the cytosolic fraction was transferred into a fresh tube, while the pellet was dissolved in $110 \mu$ of buffer B ( $250 \mathrm{mmol} / 1$ Tris-HCL, $1 \mathrm{mmol} / \mathrm{l}$ EDTA, $0.25 \mathrm{mmol} / 1$ EGTA, 2\% Triton X-100) using a homogeniser. A second centrifugation step $\left(100,000 \mathrm{~g}, 1 \mathrm{~h}\right.$ at $\left.4^{\circ} \mathrm{C}\right)$ was performed, and the supernatant (membrane fraction) was collected [16]. Proteins concentrations were determined using the BCA (bicinchoninic acid) Assay Kit (Thermo Fisher Scientific, Waltham, MA, USA). Aliquots of $30 \mu \mathrm{g}$ of total proteins, as well as cytosolic and membrane fractions, were loaded onto an SDS-polyacrylamide gradient gel (4-20\% Mini-PROTEAN TGX Precast Protein Gels 190; Bio-Rad, CA, USA) and electrophoresed. After blocking, the membranes were incubated with primary antibodies diluted $1: 1000$, if not differently specified, in combination with the respective horseradish peroxidase (HRP)-conjugated secondary anti-rabbit antibody, diluted 1:2500, or anti-mouse, diluted 1:1000. Primary antibodies, purchased from Cell Signaling Technology (Danvers, MA, USA), were as follows: Akt (9272); serine ${ }^{473}$-phosphorylation of Akt (9271); serine ${ }^{1101}$ phosphorylation of IRS-1 (2385); stress-activated protein kinase (SAPK)/ c-Jun N-terminal kinase (JNK) (9252); threonine $^{183}$-phosphorylation and thyrosine ${ }^{185}$-phosphorylation of SAPK/JNK (9251); PP2A subunit (2041); aPKCל (9372); and GAPDH $(2118 ; 1: 5000)$ as housekeeping protein for the soluble and cytosolic fractions. $\mathrm{nPKC} \theta$ (610090) and $\mathrm{nPKC} \varepsilon$ (610086) were obtained from BD Biosciences, IRS-1 (06248) from Millipore, and $\mathrm{Na}^{+} / \mathrm{K}^{+}$-ATPase, as housekeeping protein for membrane fractions, from Abcam (Ab76020; 1:10,000). Proteins were detected using a Bio-Rad 
ChemiDoc MP Imaging System in combination with ImageLab 6.0.1 software (Bio-Rad 199 Laboratories) for densitometric analysis. Data are expressed in arbitrary units and normalised to housekeeping protein.

Mitochondrial content and function Mitochondrial respiration was assessed by high-resolution respirometry (Oxygraph $2 \mathrm{k}$, O2k; Oroboros instruments, Innsbruck, Austria) in freshly harvested permeabilised muscle fibres after applying sequential substrate-uncoupler-inhibitor protocols as described elsewhere [17]. $\mathrm{H}_{2} \mathrm{O}_{2}$ emission from permeabilised muscle fibres was quantified by high-resolution respirometry with Amplex Red as previously described [17]. Citrate synthase activity (CSA) was measured spectrophotometrically (CSA Kit; Sigma-Aldrich) as a surrogate marker for mitochondrial content. Mitochondrial respiration and $\mathrm{H}_{2} \mathrm{O}_{2}$ emission were normalised to individual CSA values to account for differences in mitochondrial content.

Circulating metabolites and hormones Plasma concentrations of insulin, gastric inhibitory polypeptide (GIP), glucagon-like peptide-1 (GLP-1), glycerol, glucagon, NEFA, cortisol, alanine aminotransferase (ALT), aspartate aminotransferase (AST), triacylglycerols and chylomicrons as well as blood concentration of glucose and $\mathrm{HbA}_{1 \mathrm{c}}$ were measured as described elsewhere [11]. Plasma glycerol was measured enzymatically (r-Biopharm, Darmstadt, Germany). Serum concentrations of IL- 6 and TNF- $\alpha$ were quantified using the respective Quantikine HS ELISA kits (R\&D Systems/ BioTechne, Wiesbaden, Germany) [11].

${ }^{2} \mathrm{H}$-labelled glucose Measurement of blood $\left[{ }^{2} \mathrm{H}_{2}\right]$ glucose atom percent enrichment was performed on a Hewlett Packard 6890 gas chromatograph equipped with a $25 \mathrm{~m}$ CPSil5CB capillary column ( $0.2 \mathrm{~mm}$ i. d., $0.12 \mu \mathrm{m}$ film thickness; Chrompack/ Varian, Middelburg, the Netherlands) and interfaced to a Hewlett Packard 5975 mass selective detector [11].

Calculations During basal and clamp periods, whole-body rate of glucose disposal $\left(R_{\mathrm{d}}\right)$ was calculated from $\left[{ }^{2} \mathrm{H}_{2}\right]$ glucose enrichments (Steele's steady-state equation). During the basal period, $R_{\mathrm{d}}$ was given as $R_{\mathrm{d}}$ divided by the mean plasma insulin levels of the last $30 \mathrm{~min}$ of the respective period $(+330 \mathrm{~min}$ to $+360 \mathrm{~min})$ [10]. Endogenous glucose production (EGP) was calculated from the rate of appearance $\left(R_{\mathrm{a}}\right)$ and was expressed as EGP $\times$ insulin levels of the last $30 \mathrm{~min}$ of basal period ( $+330 \mathrm{~min}$ to $+360 \mathrm{~min})$ [18]. During the steady-state clamp period $(+450 \mathrm{~min}$ to +480 min), EGP suppression (in \%) was calculated to estimate hepatic insulin sensitivity [10]. Incremental areas under the curve (iAUCs) were calculated (basal and clamp period combined) using the trapezoidal rule corrected for the respective AUC [10].
Statistics The power calculation is based on a previous study on oral lipid-induced insulin resistance, using two simultaneous two-sided paired $t$ tests, resulting in a sample size of $n=16$ with a multiplicity adjusted $\alpha$ of 0.025 and a power of $85 \%$ [5]. Results are presented as means \pm SEM (figures), means \pm SD for normally distributed variables or median with IQR (first to third quartile) for log normally distributed variables (ESM Table 1) and compared by mixed model repeated measures ANOVA adjusted to BMI, age and sex and with Tukey-Kramer correction. Comparison of changes within one participant was done using a two-side paired $t$ test. Variables with skewed distributions were $\log _{e}$-transformed before analysis. Statistical significance of differences was defined at $p<0.05$. Calculations were performed using SAS version 9.4 (SAS Institute).

\section{Results}

PAL and SAF similarly increase plasma chylomicrons but only PAL raises palmitic acid concentrations Time-dependent changes were analysed by comparing the iAUC. After both interventions, plasma chylomicrons similarly increased by $\sim 65 \%$ from the pre-basal period $(p=0.006$ for PAL vs VCL; $p=0.010$ for SAF vs VCL; Fig. 2a). Likewise, plasma triacylglycerols increased by $\sim 37 \%$ and $28 \%$ after PAL and SAF, respectively $(p=0.004$ for PAL vs VCL; $p=0.002$ for SAF vs VCL; Fig. 2b). Plasma total NEFA were $13 \%$ and $24 \%$ higher after PAL compared with SAF or VCL, respectively ( $p=0.0008$ and $p=0.0005$; Fig. 2c). During the basal period, after PAL, plasma palmitic acid was $\sim 80 \%$ higher than after SAF or VCL ( $p=0.0010$ and $p=0.0005$, respectively; Fig. $2 \mathrm{~d}$ ). Plasma oleic acid increased by $\sim 75 \%$ after SAF and by $\sim 54 \%$ after PAL, compared with VCL $(p=0.022$ and $p$ $=0.042$, respectively; Fig. 2e). Plasma linoleic acid rose by $\sim 82 \%$ after SAF and by $\sim 78 \%$ after PAL, compared with VCL ( $p=0.001$ and $p=0.036$, respectively; Fig. 2f). There were a few differences for other NEFA species at certain time points, but the respective iAUCs for the PAL and SAF interventions were comparable (ESM Table 2).

PAL and SAF raise plasma glucagon and GIP but only SAF raises plasma GLP-1 Time-dependent changes were analysed by comparing the iAUC of the respective parameters. From the pre-basal period, plasma GIP increased eightfold and fivefold after SAF and PAL, respectively $(p=0.009$ and $p=$ 0.012 vs VCL; Fig. 3a). After SAF, plasma GLP-1 increased by sixfold and 2.5 -fold compared with VCL and PAL, respectively ( $p=0.001$ and $p=0.039 ; p=0.071$ for PAL vs VCL; Fig. 3b). During the basal period, plasma glucagon was $\sim 25 \%$ and $\sim 20 \%$ higher after PAL and SAF, respectively, compared 
Fig. 2 Time courses of circulating lipid metabolites in healthy humans. Plasma concentrations of chylomicrons (a), triacylglycerol (b), total NEFA (c), palmitic acid (d), oleic acid (e) and linoleic acid (f) after ingestion of PAL (red), SAF (blue) or VCL (water, grey) at 0 min. Data are shown as means \pm SEM; $n=16$ (chylomicrons $n=$ 10). $* p<0.05, * * p<0.01$ and $* * * p<0.001$ vs VCL at same time point; ${ }^{\dagger} p<0.05$ for PAL vs SAF at same time point (ANOVA adjusted for repeated measures with Tukey-Kramer correction for each time point between interventions). P-basal, pre-basal
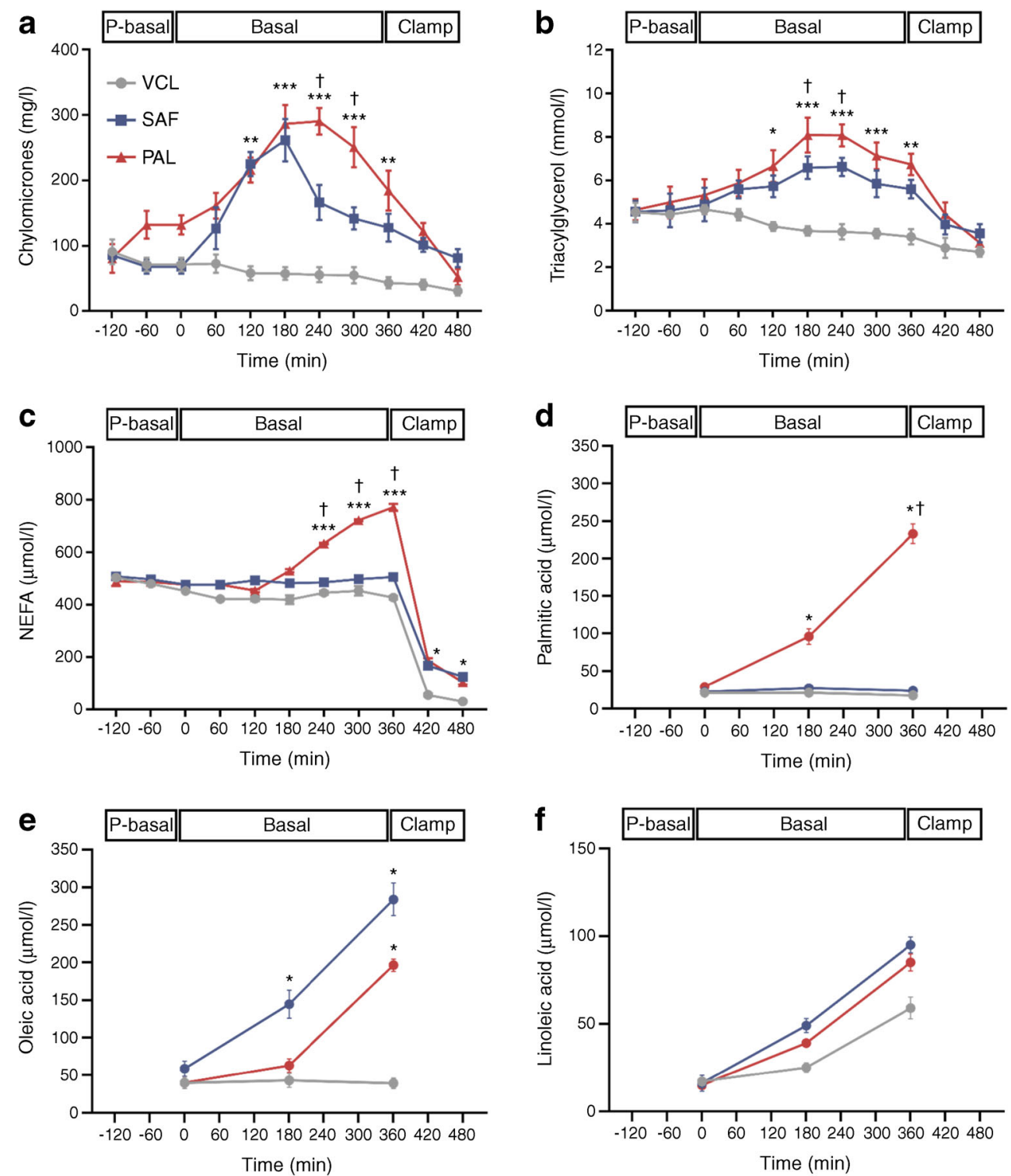

with VCL ( $p=0.021$ and $p=0.044$; Fig. $3 \mathrm{c}$ ). Plasma insulin and blood glucose did not differ between the interventions (Fig. 3d,e). Plasma glycerol decreased (by $\sim 30 \%$ from prebasal period), only after PAL, compared with $\operatorname{VCL}(p=$ 0.041; Fig. 3f).

\section{PAL and SAF lead to whole-body insulin resistance during} postprandial hyperinsulinaemia During the basal period, whole-body resting energy expenditure (REE) (PAL $7317 \pm$ $309 \mathrm{~kJ} /$ day; SAF $7606 \pm 267 \mathrm{~kJ} /$ day; VCL $7296 \pm 259 \mathrm{~kJ} /$ day), rates of lipid oxidation (LOX) (PAL $1.0 \pm$ $0.1 \mathrm{mg} \mathrm{kg}^{-1} \mathrm{~min}^{-1}$; SAF $1.1 \pm 0.2 \mathrm{mg} \mathrm{kg}^{-1} \mathrm{~min}^{-1}$; VCL $1.1 \pm 0.1 \mathrm{mg} \mathrm{kg}^{-1} \mathrm{~min}^{-1}$ ) and glucose oxidation (GOX) $\left(\mathrm{PAL} 2.2 \pm 0.2 \mathrm{mg} \mathrm{kg}^{-1} \mathrm{~min}^{-1} ; \mathrm{SAF} 2.1 \pm\right.$ $0.5 \mathrm{mg} \mathrm{kg}^{-1} \mathrm{~min}^{-1}$; VCL $2.1 \pm 0.2 \mathrm{mg} \mathrm{kg}^{-1} \mathrm{~min}^{-1}$ ) were not different between the interventions. Only after PAL, whole-body glucose disappearance $\left(R_{\mathrm{d}}\right.$ /insulin) was $28 \%$ lower $(p=0.036$; Fig. $4 \mathrm{a})$ and EGP $\times$ insulin was $38 \%$ higher compared with VCL ( $p=0.009$; Fig. $4 b$ ).

During the steady-state of the clamp, reflecting postprandial hyperinsulinaemia (Fig. 3d and ESM Table 3), REE was also comparable between the interventions (PAL $7556 \pm$ $330 \mathrm{~kJ} /$ day; SAF $7543 \pm 305 \mathrm{~kJ} /$ day; VCL $7368 \pm 284 \mathrm{~kJ} /$ day). After PAL and SAF, LOX was $85 \%$ and $70 \%$ higher compared with VCL, respectively (PAL $3.4 \pm$ $0.9 \mathrm{mg} \mathrm{kg}^{-1} \min ^{-1}[p=0.013 \mathrm{vs}$ VCL $]$; SAF $2.0 \pm$ $0.7 \mathrm{mg} \mathrm{kg}^{-1} \min ^{-1}[p=0.019$ vs VCL]; VCL $0.5 \pm$ $0.2 \mathrm{mg} \mathrm{kg}^{-1} \mathrm{~min}^{-1}$ ). Insulin-stimulated $R_{\mathrm{d}}$ was $49 \%$ and $36 \%$ lower after PAL and SAF, respectively, compared with VCL $(p<0.0001$ and $p=0.0002$; Fig. $4 \mathrm{c}$ ), partly due to $69 \%$ and $76 \%$ lower GOX after PAL and SAF $(p=0.003$ and $p=$ 0.004 vs VCL, respectively; Fig. 4d). Further, insulin- 
Fig. 3 Time courses of circulating hormones and metabolites in healthy humans. Concentrations of plasma GIP (a), plasma GLP-1 (b), plasma glucagon (c), plasma insulin (d), blood glucose (e) and plasma glycerol (f) are presented after ingestion of PAL (red), SAF (blue) or VCL (water, grey) at 0 min. Data are shown as means \pm SEM; insulin and blood glucose $n=16$; GIP, GLP-1, glucagon and glycerol, $n=4 . * p<0.05$, $* * p<0.01$ and $* * * p<0.001 \mathrm{vs}$ VCL at same time point; ${ }^{\dagger} p<0.05$ and ${ }^{\dagger \dagger} p<0.01$ for PAL vs SAF at same time point (ANOVA adjusted for repeated measures with Tukey-Kramer correction for each time point between interventions). P-basal, pre-basal
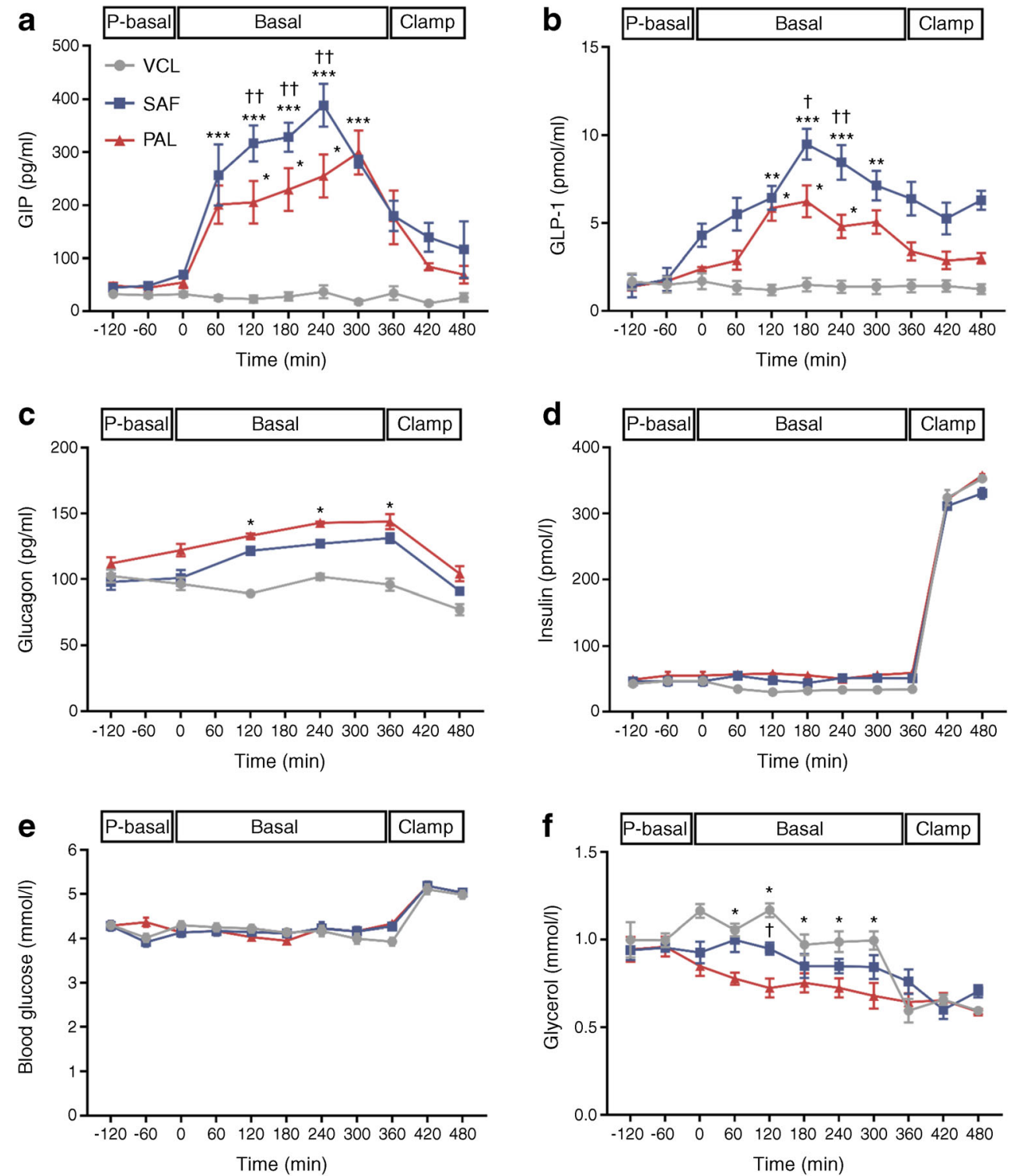

stimulated $R_{\mathrm{d}}$ was $13 \%$ lower after PAL than after SAF ( $p$ $=0.041)$, due to $59 \%$ lower non-oxidative glucose disposal (NOXGD) ( $p=0.008$ vs SAF; Fig. 4e). Hepatic insulin resistance was $39 \%$ and $24 \%$ lower after PAL and SAF $(p<0.0001$ and $p=0.0010$ vs VCL, respectively; Fig. 4f). No sex differences were found between interventions (ESM Fig. 3).

PAL and SAF associate with the DAG-nPKC pathway Timedependent changes were analysed by comparing the iAUC of the respective parameters. Across basal and clamp periods combined, the concentration of $18-1: 18-1 ; 16-$ $0: 16-0 ; 16-0: 18-1$ DAG species in the membrane compartment was increased by $\sim 43 \%$ ( $p=0.032$ ) after PAL and by $\sim 30 \%$ ( $p=0.041$ ) after SAF (Fig. 5a). The accumulation of these DAG species in lipid droplets increased by $\sim 25 \%$ after PAL only ( $p=0.034$ vs VCL; $p=0.122$ vs SAF; Fig. $5 b)$. Membrane translocation of $\mathrm{nPKC} \varepsilon$ increased by $75 \%$ after PAL ( $p=0.003$ vs VCL; $p<0.0001$ vs SAF; Fig. 5c) and tended to rise after SAF but did not reach statistical significance ( $p=0.061$ vs VCL). Activation of $\mathrm{nPKC} \theta$ was $94 \%$ higher with PAL ( $p=0.004$ vs VCL; $p=$ 0.009 vs SAF) and $31 \%$ higher with $\operatorname{SAF}(p=0.041$ vs VCL; Fig. 5d). Myocellular serine ${ }^{1101}$-phosphorylation of IRS- 1 was increased by $57 \%$ and $52 \%$ upon PAL and SAF ingestion $(p=0.037$ and $p=0.039$, respectively, vs VCL; Fig. 5e, f).

PAL further associates with the ceramide-aPKCC-PP2A pathway Time-dependent changes were analysed by comparing 
Fig. 4 Rates of whole-body glucose disposal $\left(R_{\mathrm{d}}\right)$ and EGP during basal and clamp periods in healthy humans. (a, b) During the last 30 min of basal period $(+330 \mathrm{~min}$ to $+360 \mathrm{~min})$, rates of glucose metabolism are presented in the context of the ambient plasma insulin concentration: $R_{\mathrm{d}} /$ insulin (a) and EGP $\times$ insulin (b). (c-f) During clamp steady-state $(+450 \mathrm{~min}$ to +480 min), insulin-stimulated $R_{\mathrm{d}}(\mathbf{c})$, rate of GOX $(\mathbf{d})$, rate of NOXGD (e) and EGP suppression (f) are presented after PAL (red), SAF (blue) or VCL (water, grey) ingestion at 0 min. Data are shown as means \pm SEM; $n=16$. $* p<0.05, * * p<0.01$ and $* * * p<0.001$ vs VCL; ${ }^{\dagger} p<0.05$ and ${ }^{\dagger \dagger} p<0.01$ for PAL vs SAF (ANOVA adjusted for repeated measures with Tukey-Kramer correction)
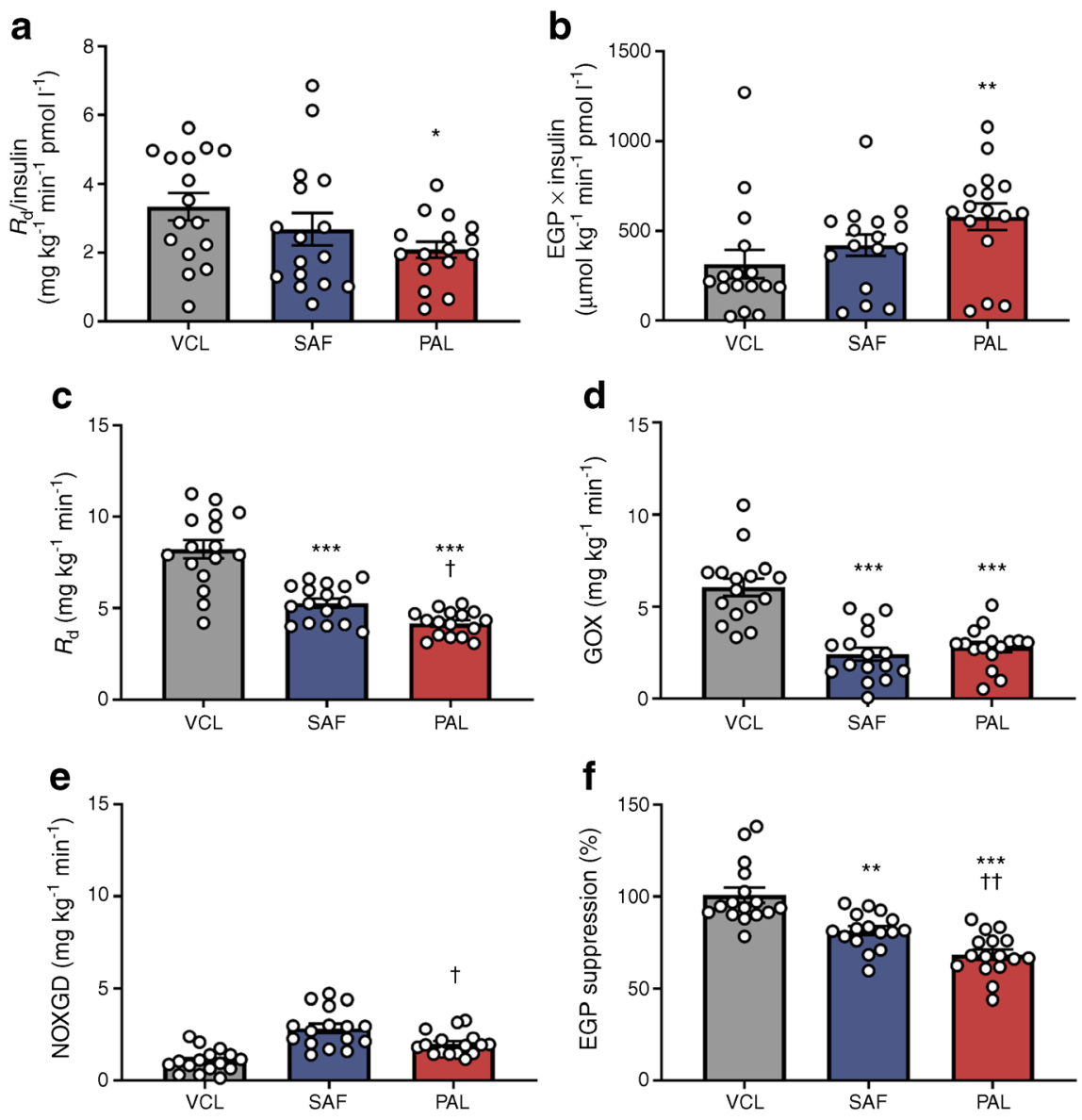

the iAUC of the respective parameters. After PAL only, concentrations of 16:0;18:0;18:1 ceramide species increased in the membrane compartment, compared with after VCL ( $\sim 30 \%$ increase, $p=0.022$ for PAL vs VCL; $p=0.078$ for SAF vs VCL; Fig. 6a and ESM Table 4). Similarly, concentrations of these ceramide species in lipid droplets were $\sim 30 \%$ and $\sim 20 \%$ higher after PAL than after VCL or SAF ( $p=$ 0.017 PAL vs VCL; $p=0.025$ PAL vs SAF; Fig. 6b). Membrane-to-cytosol translocation of aPKC $\zeta$ was $\sim 69 \%$ and $\sim 59 \%$ higher after PAL than after VCL $(p=0.013)$ or SAF ( $p=0.035$; Fig. $6 \mathrm{c}$ ), respectively. In addition, PAL induced a $\sim 35 \%$ higher myocellular PP2A expression, when compared with VCL $(p=0.031)$ or SAF ( $p=0.039$; Fig. 6d). Only after PAL, serine ${ }^{473}$-phosphorylation of Akt was reduced by $40 \%$ and $36 \%$ compared with VCL $(p=0.022)$ or SAF ( $p=0.034$; Fig. $6 \mathrm{e}, \mathrm{f}$ ), respectively.

PAL and SAF do not acutely affect mitochondrial oxidative capacity During the basal period, SAF and PAL, compared with VCL ingestion, had no effect on total muscle CSA, maximum coupled and uncoupled mitochondrial oxidative capacity from succinate and carbonyl cyanide $p$-trifluoromethoxyphenyl hydrazine (FCCP) normalised to CSA, $\beta$ oxidation from octanoyl-carnitine related to $\mathrm{CSA}, \mathrm{H}_{2} \mathrm{O}_{2}$ emission normalised to CSA and leak control ratio, which reflects increased proton leak across the mitochondrial membrane (ESM Fig. 4a-f).

PAL and SAF do not acutely affect biomarkers of inflammation Variables of inflammation in the circulation (IL-6, TNF- $\alpha$, cortisol) and in skeletal muscle (c-JNK, phosphorylated JNK) did not differ between interventions during basal and clamp periods (ESM Table 5).

\section{Discussion}

This study shows that a single lipid load, regardless of its FA composition, causes whole-body and hepatic insulin resistance. Palmitate-rich lipid ingestion was associated with activation of the myocellular DAG-nPKC pathway, leading to inhibitory serine ${ }^{1101}$-phosphorylation of IRS-1, already during basal insulinaemia. Of note, PAL also increased myocellular ceramide content, which may contribute to skeletal muscle insulin resistance via activation of $\mathrm{aPKC} \zeta$ and PP2A and inhibition of Akt. Furthermore, neither palmitate- nor oleatecontaining lipid loads acutely affected mitochondrial oxidative capacity or inflammatory pathways in human skeletal muscle. 
Fig. 5 Myocellular lipid metabolites and insulin signalling (DAG-nPKC pathway) in healthy humans. DAG species $18-1: 18-1,16-0: 16-0$ and 16 $0: 18-1$ in the cell membrane fraction (a), DAG species 18$1: 18-1,16-0: 16-0$ and 16-0:18 1 in the lipid droplet fraction (b), $\mathrm{nPKC} \varepsilon$ activation $(\mathbf{c}), \mathrm{nPKC} \theta$ activation (d) and IRS-1 levels (e) as well as serine ${ }^{1101}$ -

phosphorylation of IRS-1 relative to IRS-1 (f) during the pre-basal, basal and clamp periods after ingestion of PAL (red), SAF (blue) or VCL (water, grey) at 0 min. Expression signals on immunoblots are expressed in arbitrary units (AU) after normalising against GAPDH for total and cytosolic proteins and against $\mathrm{Na}^{+} / \mathrm{K}^{+}$-ATPase for membrane proteins. Data are shown as means $\pm \mathrm{SEM} ; n=$ 16 at time point $-60 \mathrm{~min}, n=$ 10 at +120 min, $n=6$ at +240 min and +420 min. $* p<$ $0.05 \mathrm{vs}$ VCL at same time point (ANOVA adjusted for repeated measures with Tukey-Kramer correction for each time point between interventions). LD, lipid droplet fraction; P-basal, prebasal
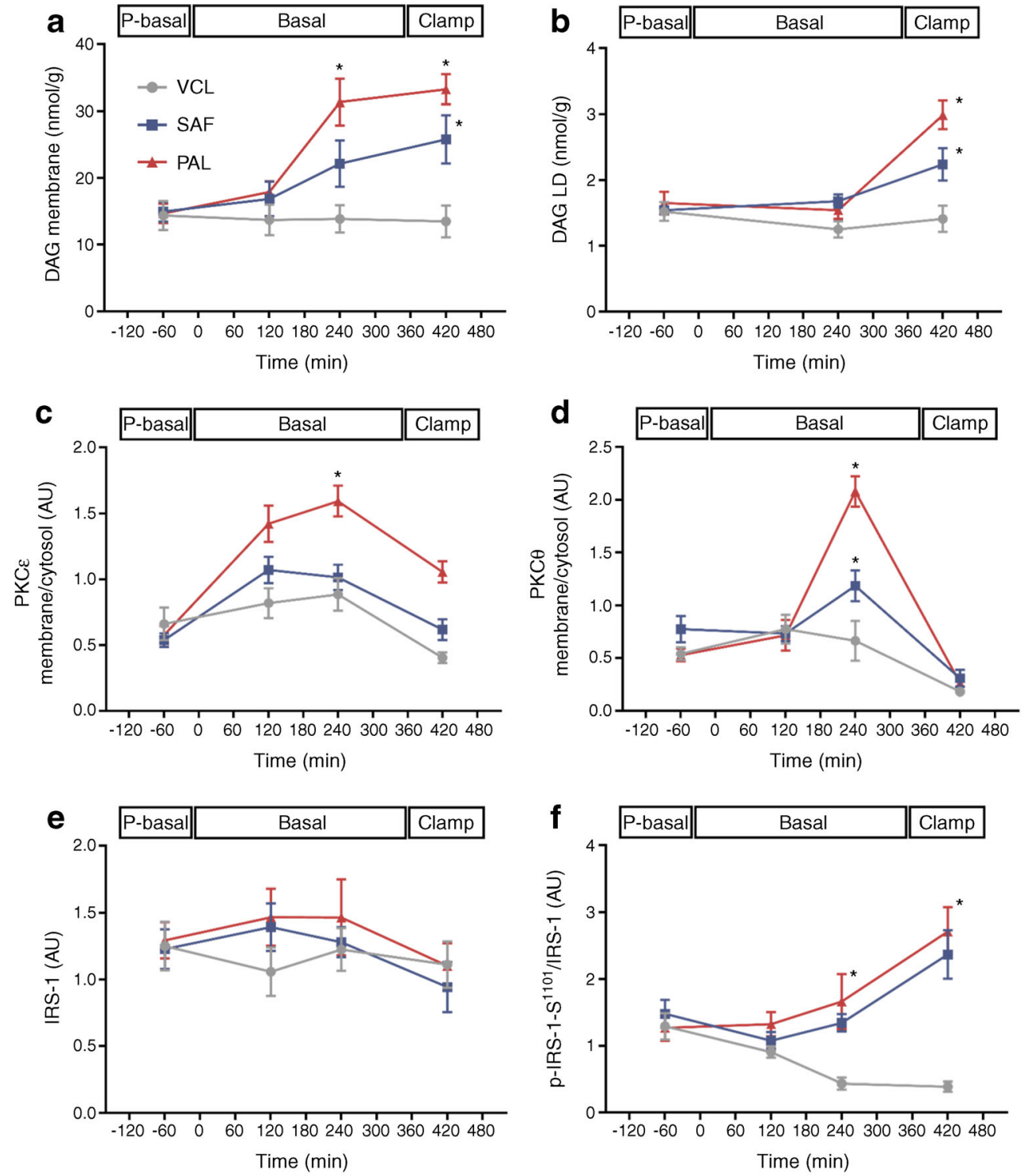

Both SAF (enriched in oleate) and PAL (enriched in palmitate and oleate) decreased whole-body insulin sensitivity under hyperinsulinaemic conditions. Compared with SAF, PAL had a somewhat more pronounced inhibitory effect on insulin sensitivity during the clamp test, with reduction in insulin-stimulated NOXGD as well as glucose disposal during the basal period of fasting insulinaemia, as seen in most $[5,11]$ but not all human studies [12]. Of note, lipid loading may cause substantial substrate competition, particularly in the absence of insulin stimulation. In the present study, SAF and PAL did not seem to affect the LOX/GOX ratio during the basal period and LOX was only nominally higher under clamp conditions. Taken together, both lipid interventions markedly decreased insulin-stimulated whole-body glucose disposal, at least partly due to NOXGD, which mainly reflects skeletal muscle insulin resistance.
So far, most controlled human studies investigating lipidinduced insulin resistance have used i.v. or oral mixed lipid challenges without distinguishing between the degrees of FA saturation [19]. The present study now extends these previous results $[5,6,19,20]$ in that both single lipid meals, but especially PAL already during the basal period, acutely increased membrane $\mathrm{C} 18$-containing DAG species, followed by $\mathrm{nPKC} \theta$ translocation, serine ${ }^{1101}$-phosphorylation of IRS-1 and wholebody insulin resistance. This sequence of events has been also demonstrated for i.v. infusion of lipids and supports the causal relationship observed in in vitro studies [21]. The subsequent decrease in $n P K C \theta$ during the clamp could be due to insulindependent lowering of intracellular FA-CoA levels, which may synergistically activate nPKCs [22]. Later in the course of study, DAG species also accumulated in the lipid droplet compartment, likely reflecting elevated triacylglycerol 
Fig. 6 Myocellular lipid metabolites and insulin signalling (ceramide-aPKC pathway) in healthy humans. Ceramide species 16:0, 18:0 and 18:1 in the cell membrane fraction (a), ceramide species 16:0, 18:0 and 18:1 in the lipid droplet fraction (b), aPKC $\zeta$ activation (c), PP2A (d), Akt (e) and serine ${ }^{473}$ phosphorylation of Akt relative to Akt (f) after ingestion of PAL (red), SAF (blue) or VCL (water, grey) at 0 min. Expression signals on immunoblots are expressed in arbitrary units (AU) after normalising against GAPDH for total and cytosolic proteins and against $\mathrm{Na}^{+} / \mathrm{K}^{+}$-ATPase for membrane proteins. Data are shown as means \pm SEM; $n=$ 16 at time point $-60 \mathrm{~min}, n=$ 10 at $+120 \min , n=6$ at $+240 \mathrm{~min}$ and +420 min. *p < 0.05 vs VCL at same time point; ${ }^{\dagger} p<0.05$ for PAL vs SAF at same time point (ANOVA adjusted for repeated measures with Tukey-Kramer correction for each time point between interventions). P-basal; pre-basal
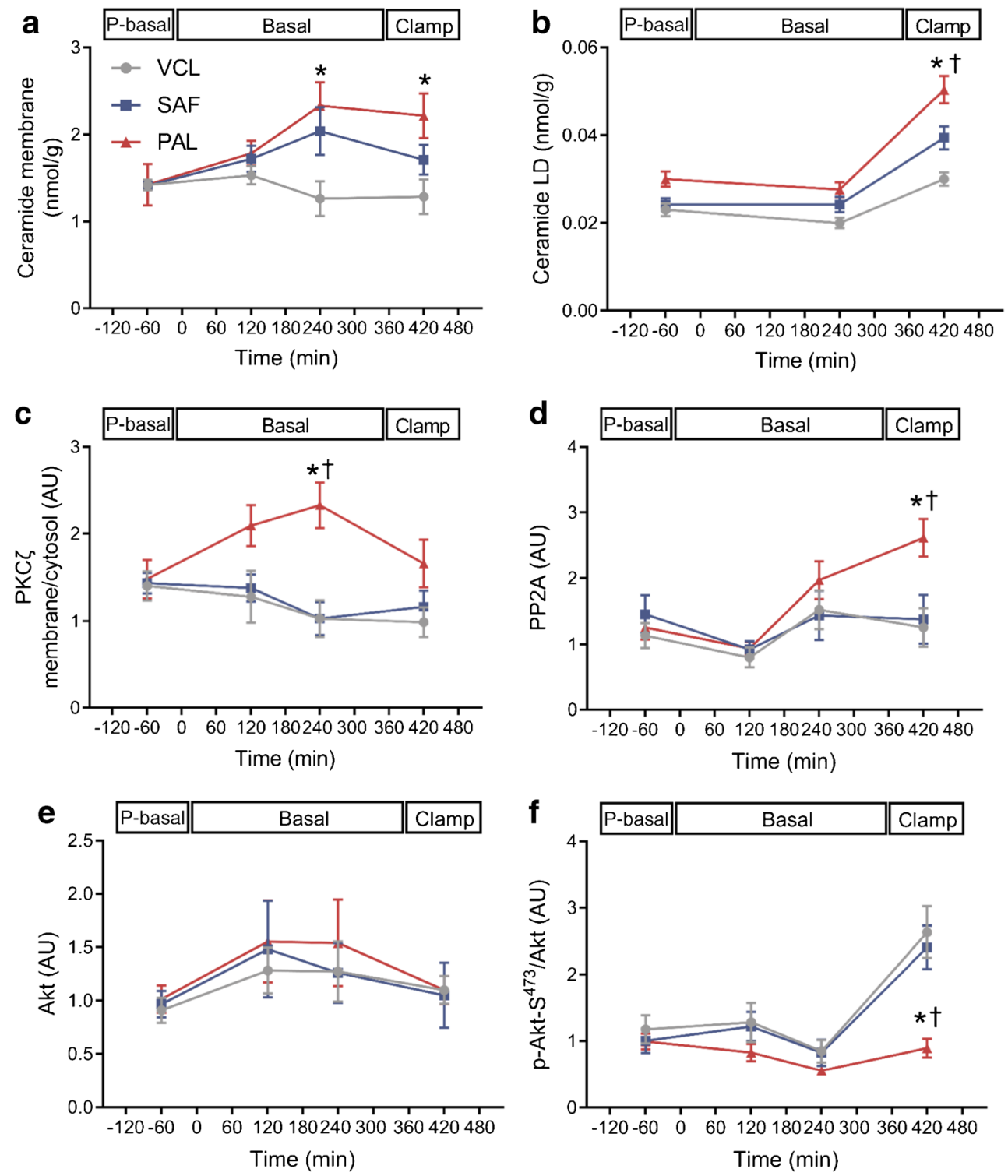

synthesis. Of note, the present study did not further analyse the C-18-containing DAG for its 1,2 stereoisomers, which are the bioactive mediators of insulin resistance [1, 20]. In line, previous studies indicate that palmitate exposure can induce higher myocellular DAG production than oleate [23, 24], associated with lower insulin-stimulated myocellular glucose uptake [25]. Furthermore, we observed increased nPKCE translocation after PAL but not SAF. Previous studies have linked lipid-mediated insulin resistance mainly to $\mathrm{nPKC} \theta$ activation in rodents [26] and humans $[5,6]$. However, higher skeletal muscle $\mathrm{nPKC} \varepsilon$ activity was demonstrated in obesity or type 2 diabetes, compared with lean humans, as well [20].

Interestingly, only the single palmitate-rich lipid ingestion but not the oleate-rich lipid ingestion also increased the myocellular ceramide content, again first in the membrane and then in lipid droplets, the cellular lipid repository [15].
This was accompanied by activation of the aPKC $\zeta-\mathrm{PP} 2 \mathrm{~A}$ pathway [27, 28], by which membrane-bound ceramide is known to inhibit serine ${ }^{473}$-phosphorylation of Akt [29]. Exclusively, PAL decreased the myocellular serine ${ }^{473}$-phosphorylated Akt/Akt ratio during hyperinsulinaemia, indicating decreased distal insulin signalling. In an obese mouse model, inhibition of serine palmitoyl-transferase-1, an enzyme involved in de novo synthesis of ceramides from saturated FA, led to acute improvement of muscle Akt phosphorylation and insulin sensitivity [30,31]. Thus, the lack of a similar effect with SAF despite the observed insulin resistance could result from different lipotoxic synthesis pathways based on the degree of saturation of the ingested FA intervention [32]. Alternatively, higher total and other NEFA concentrations following PAL vs SAF may explain PAL's more pronounced inhibitory effect on Akt. 
Generally, saturated fat, particularly palmitate, has been associated with reduced whole-body insulin sensitivity [33]. A 7-day diet intervention in lean women but not men linked a palmitate-rich diet to higher myocellular ceramides with lower insulin sensitivity compared with an oleate-rich diet [34]. Of note, in cultured human myotubes, the addition of an equal amount of oleate suddenly improved palmitate-induced ceramide-mediated insulin resistance $[25,35]$. This mitigating effect of oleate on lipotoxicity through palmitate-induced increase in ceramides has been previously explained by augmented mitochondrial FA metabolism [36], although oleate and palmitate compared with palmitate alone were able to increase triacylglycerol storage, thereby preventing DAG accumulation and insulin resistance at least in cultured muscle cells [23].

Our study further confirms an increase in membrane C16to $\mathrm{C} 18$-ceramide species, which were acutely increased in lean and obese humans in the context of insulin resistance upon palmitate ingestion [37]. Another study found an inverse relationship between elevated myocellular ceramides and insulin sensitivity in lean men after an i.v. infusion of mixed lipids/ heparin [38]. In addition, Perreault et al reported a relationship of sarcolemmal ceramides, but not DAG, with human insulin resistance [20]. Of note, other studies found neither alterations of myocellular sphingolipids nor relationships with insulin sensitivity in people who were obese and/or had type 2 diabetes $[6,39,40]$. In addition, the finding that unsaturated FA can induce insulin resistance without elevating ceramide content suggests that the ceramide-mediated impairment of distal insulin signalling may not be mandatory for lipid-induced insulin resistance [41]. However, the observation that the palmitate-induced increase in myocellular ceramides appears to aggravate acute lipid-mediated insulin resistance when compared with oleate alone supports the hypothesis of synergic inhibition of insulin signalling by different lipid mediators, at least under conditions of a single oral lipid load [42]. Furthermore, the palmitate-enriched intervention also resulted in lower EGP during basal insulinaemia, which would favour higher rates of gluconeogenesis and lipogenesis [10, 11]. During the clamp, both SAF and, more markedly, PAL decreased hepatic insulin sensitivity. This is remarkable, as this one-step clamp was not primarily designed to examine hepatic insulin action, indicating a strong stimulatory effect on EGP, which may be explained by FA-induced allosteric stimulation of gluconeogenesis and, to a minor extent, by increased glycerol uptake, serving as gluconeogenic substrate $[1,2]$. In addition, incretin-mediated changes in portal insulin and glucagon concentrations may mask the effects of oral lipid intake on hepatic glucose metabolism [43]. Furthermore, higher GLP-1 concentrations may lead to increased NEFA uptake from plasma chylomicrons and suppression of FA spillover into the circulation [44]. This could explain the absent increase in NEFA despite elevated chylomicrons after
SAF vs PAL seen in the current study. Previous acute high-fat diet intervention studies demonstrated similar $[10,11]$ or no effects in humans [5, 45], suggesting that the different results are not due to lipid composition but rather due to study design or total energy intake.

Interestingly, the present study failed to detect changes in circulating inflammatory cytokines, cortisol or classical cellular inflammatory pathways in line with previous studies on acute effects of saturated [11] and monounsaturated FAs in humans $[5,10]$. This underlines that the known increase of low-grade inflammation occurring upon high-fat diet [34] is not involved in the acute initiation of muscle insulin resistance but rather results over time from secondary alterations, likely arising from adipose tissue dysfunction [1]. Likewise, the present study found no acute effects of PAL or SAF on muscle mitochondrial enzyme activity, oxidative capacity or $\mathrm{H}_{2} \mathrm{O}_{2}$ emission during the basal period, in line with previous studies [46], supporting the concept that lipotoxic signalling is the primary event of lipid-induced muscle insulin resistance, regardless of the lipid composition. Nevertheless, excessive mitochondrial $\mathrm{H}_{2} \mathrm{O}_{2}$ production has been linked to insulin resistance in a high-fat diet model in rodents and humans [47], indicating effects on mitochondrial function operating during long-term lipid overfeeding.

The present study benefits from the serial biopsies allowing for real-time monitoring and analyses of the sequence of cellular events in human skeletal muscle, although by design it cannot provide a definite mechanistic proof. Limitations include the use of pure oils, rather than the consumption of mixed meals. In addition, using water as control will lead to specific metabolic and endocrine conditions (e. g. substrate competition, lipolysis with endogenous NEFA spill-out [48] and low level postprandial incretins). The lipid interventions were designed to be isoenergetic $(\sim 3012 \mathrm{~kJ})$ so that differences in effects are unlikely to be due to energy intake. Also, the fact that no strict monitoring of diet and exercise was feasible on the days before the interventions might have influenced the acute lipid overload response. Moreover, the results of this short-term study in healthy humans cannot be necessarily extrapolated to chronic lipid oversupply or common insulin resistance of obesity and type 2 diabetes. Nevertheless, our approach represents an accepted compromise between optimal experimental and near-physiological conditions to allow for assessing acute metabolic changes [10-12].

In conclusion, this short-term study reveals that raising plasma oleate with and without palmitate concentrations is associated with activation of the myocellular DAG-nPKC pathway and muscle insulin resistance. The acute increase in plasma palmitate also favours ceramide accumulation and aPKC $\zeta-\mathrm{PP} 2 \mathrm{~A}$ activation, which can further aggravate muscle insulin resistance. The possible role of lipid interactions, such as modulation by oleate of the palmitate-induced inhibitory 
signalling events, remains to be investigated. All these effects seem to occur in the absence of changes in mitochondrial capacity or inflammatory pathways, underlining the relevance of lipotoxic pathways as therapeutic targets to prevent and reverse muscle insulin resistance in humans. Finally, further studies are required to prove the effects of diets containing different lipids in healthy and insulin-resistant humans.

Supplementary Information The online version contains peer-reviewed but unedited supplementary material available at https://doi.org/10.1007/ s00125-021-05596-z.

Acknowledgements We would like to thank K. Straßburger, F. Ziehve, M. Reina de Fundo, M. Eßer, D. Höhn, U. Partke and A. Sparla, staff members of the Institute of Clinical Diabetology at the German Diabetes Center, for their excellent support.

Data availability All data generated or analysed during this study are included in the published article (and its online supplementary files). The files are available from the corresponding author upon reasonable request.

Funding Open Access funding enabled and organized by Projekt DEAL. This study was supported by the German Federal Ministry of Health, the Ministry of Culture and Science of the State North Rhine-Westphalia, the German Federal Ministry of Education and Research, the European Regional Development Fund (KomIT, EFRE-0400191) and the German Research Foundation (DFG; SFB 1116/2) and supported in part by the German Center for Diabetes Research e. V.

Authors' relationships and activities MR received personal fees from Boehringer-Ingelheim Pharma, Eli Lilly, Fishawack Group, Novo Nordisk, Sanofi US, Target NASH and Terra Firma, and investigatorinitiated research support from Boehringer-Ingelheim, Nutricia/Danone and Sanofi-Aventis. $\mathrm{CH}$ is a member of the editorial board of Diabetologia. $\mathrm{CH}$ received research support from Sanofi-Aventis. All other authors declare that there are no relationships or activities that might bias, or be perceived to bias, their work.

Contribution statement MR initiated and led the clinical experiments and wrote, reviewed and edited the manuscript. TS led the clinical experiments, analysed the data and wrote and edited the manuscript. CK, SK, ACB, MW, MA, DFM, LM, DP and CH obtained and analysed data and edited the manuscript. $\mathrm{PB}$ performed statistical analysis and reviewed the manuscript. All authors have given final approval of the version to be published. MR is the guarantor of this work and, as such, had full access to all the data in the study and takes responsibility for the integrity of the data and data analysis.

Open Access This article is licensed under a Creative Commons Attribution 4.0 International License, which permits use, sharing, adaptation, distribution and reproduction in any medium or format, as long as you give appropriate credit to the original author(s) and the source, provide a link to the Creative Commons licence, and indicate if changes were made. The images or other third party material in this article are included in the article's Creative Commons licence, unless indicated otherwise in a credit line to the material. If material is not included in the article's Creative Commons licence and your intended use is not permitted by statutory regulation or exceeds the permitted use, you will need to obtain permission directly from the copyright holder. To view a copy of this licence, visit http://creativecommons.org/licenses/by/4.0/.

\section{References}

1. Roden M, Shulman GI (2019) The integrative biology of type 2 diabetes. Nature 576(7785):51-60. https://doi.org/10.1038/s41586019-1797-8

2. Echouffo-Tcheugui JB, Ahima RS (2019) Does diet quality or nutrient quantity contribute more to health? J Clin Invest 129(10): 3969-3970. https://doi.org/10.1172/JCI131449

3. Piepoli MF, Hoes AW, Agewall S et al (2016) 2016 European guidelines on cardiovascular disease prevention in clinical practice: the sixth joint task force of the European Society of Cardiology and Other Societies on cardiovascular disease prevention in clinical practice (constituted by representatives of 10 societies and by invited experts) developed with the special contribution of the European Association for Cardiovascular Prevention \& rehabilitation (EACPR). Eur Heart J 37(29):2315-2381. https://doi.org/10.1093/ eurheartj/ehw106

4. American Diabetes Association (2018) 4. Lifestyle management: standards of medical Care in Diabetes-2018. Diabetes Care 41(Suppl 1):S38-s50. https://doi.org/10.2337/dc18-S004

5. Nowotny B, Zahiragic L, Krog D et al (2013) Mechanisms underlying the onset of oral lipid-induced skeletal muscle insulin resistance in humans. Diabetes 62:2240-2248. https://doi.org/10.2337/ db12-1179

6. Szendroedi J, Yoshimura T, Phielix E et al (2014) Role of diacylglycerol activation of PKCtheta in lipid-induced muscle insulin resistance in humans. Proc Natl Acad Sci U S A 111(26):95979602. https://doi.org/10.1073/pnas.1409229111

7. Kim JK, Fillmore JJ, Sunshine MJ et al (2004) PKC-theta knockout mice are protected from fat-induced insulin resistance. J Clin Invest 114(6):823-827. https://doi.org/10.1172/jci22230

8. Tripathy D, Mohanty P, Dhindsa S et al (2003) Elevation of free fatty acids induces inflammation and impairs vascular reactivity in healthy subjects. Diabetes 52(12):2882-2887. https://doi.org/10. 2337/diabetes.52.12.2882

9. Dubé JJ, Coen PM, DiStefano G et al (2014) Effects of acute lipid overload on skeletal muscle insulin resistance, metabolic flexibility, and mitochondrial performance. Am J Physiol Endocrinol Metab 307(12):E1117-E1124. https://doi.org/10.1152/ajpendo.00257. 2014

10. Sarabhai T, Kahl S, Szendroedi J et al (2020) Monounsaturated fat rapidly induces hepatic gluconeogenesis and whole-body insulin resistance. JCI Insight 5(10):e134520. https://doi.org/10.1172/jci. insight. 134520

11. Hernandez EA, Kahl S, Seelig A et al (2017) Acute dietary fat intake initiates alterations in energy metabolism and insulin resistance. J Clin Invest 127(2):695-708. https://doi.org/10.1172/ JCI89444

12. Xiao C, Giacca A, Carpentier A, Lewis GF (2006) Differential effects of monounsaturated, polyunsaturated and saturated fat ingestion on glucose-stimulated insulin secretion, sensitivity and clearance in overweight and obese, non-diabetic humans. Diabetologia 49(6):1371-1379. https://doi.org/10.1007/s00125006-0211-x 
13. Gao D, Griffiths HR, Bailey CJ (2009) Oleate protects against palmitate-induced insulin resistance in L6 myotubes. Br J Nutr 102(11):1557-1563. https://doi.org/10.1017/s0007114509990948

14. Lepage G, Roy CC (1986) Direct transesterification of all classes of lipids in a one-step reaction. J Lipid Res 27(1):114-120. https://doi. org/10.1016/S0022-2275(20)38861-1

15. Preuss C, Jelenik T, Bódis K et al (2019) A new targeted Lipidomics approach reveals lipid droplets in liver, muscle and heart as a repository for diacylglycerol and ceramide species in non-alcoholic fatty liver. Cells 8(3):277. https://doi.org/10.3390/ cells 8030277

16. Apostolopoulou M, Mastrototaro L, Hartwig S et al (2021) Metabolic responsiveness to training depends on insulin sensitivity and protein content of exosomes in insulin resistant males. Sci Adv 7(41):eabi9551. https://doi.org/10.1126/sciadv.abi9551.

17. Phielix E, Jelenik T, Nowotny P, Szendroedi J, Roden M (2014) Reduction of non-esterified fatty acids improves insulin sensitivity and lowers oxidative stress, but fails to restore oxidative capacity in type 2 diabetes: a randomised clinical trial. Diabetologia 57(3):572581. https://doi.org/10.1007/s00125-013-3127-2

18. Hattersley JG, Möhlig M, Roden M et al (2012) Quantifying the improvement of surrogate indices of hepatic insulin resistance using complex measurement techniques. PLoS One 7(6):e39029. https:// doi.org/10.1371/journal.pone.0039029

19. Itani SI, Ruderman NB, Schmieder F, Boden G (2002) Lipidinduced insulin resistance in human muscle is associated with changes in diacylglycerol, protein kinase $\mathrm{C}$, and IkappaB-alpha. Diabetes 51(7):2005-2011. https://doi.org/10.2337/diabetes.51.7. 2005

20. Perreault L, Newsom SA, Strauss A et al (2018) Intracellular localization of diacylglycerols and sphingolipids influences insulin sensitivity and mitochondrial function in human skeletal muscle. JCI Insight 3(3):e96805. https://doi.org/10.1172/jci.insight.96805

21. Li Y, Soos TJ, Li X et al (2004) Protein kinase C Theta inhibits insulin signaling by phosphorylating IRS1 at Ser(1101). J Biol Chem 279(44):45304-45307. https://doi.org/10.1074/jbc. C400186200

22. Idris I, Gray S, Donnelly R (2001) Protein kinase C activation: isozyme-specific effects on metabolism and cardiovascular complications in diabetes. Diabetologia 44(6):659-673. https://doi.org/10. $1007 / \mathrm{s} 001250051675$

23. Gaster M, Rustan AC, Beck-Nielsen H (2005) Differential utilization of saturated palmitate and unsaturated oleate: evidence from cultured myotubes. Diabetes 54(3):648-656. https://doi.org/10. 2337/diabetes.54.3.648

24. Bergman BC, Hunerdosse DM, Kerege A, Playdon MC, Perreault L (2012) Localisation and composition of skeletal muscle diacylglycerol predicts insulin resistance in humans. Diabetologia 55(4): 1140-1150. https://doi.org/10.1007/s00125-011-2419-7

25. Montell E, Turini M, Marotta M et al (2001) DAG accumulation from saturated fatty acids desensitizes insulin stimulation of glucose uptake in muscle cells. Am J Physiol Endocrinol Metab 280(2): E229-E237. https://doi.org/10.1152/ajpendo.2001.280.2.E229

26. Griffin ME, Marcucci MJ, Cline GW et al (1999) Free fatty acidinduced insulin resistance is associated with activation of protein kinase $\mathrm{C}$ theta and alterations in the insulin signaling cascade. Diabetes 48(6):1270-1274. https://doi.org/10.2337/diabetes.48.6. 1270

27. Stratford S, Hoehn KL, Liu F, Summers SA (2004) Regulation of insulin action by ceramide: dual mechanisms linking ceramide accumulation to the inhibition of Akt/protein kinase B. J Biol
Chem 279(35):36608-36615. https://doi.org/10.1074/jbc. M406499200

28. Blouin CM, Prado C, Takane KK et al (2010) Plasma membrane subdomain compartmentalization contributes to distinct mechanisms of ceramide action on insulin signaling. Diabetes 59(3): 600-610. https://doi.org/10.2337/db09-0897

29. Schmitz-Peiffer C, Craig DL, Biden TJ (1999) Ceramide generation is sufficient to account for the inhibition of the insulin-stimulated $\mathrm{PKB}$ pathway in $\mathrm{C} 2 \mathrm{C} 12$ skeletal muscle cells pretreated with palmitate. J Biol Chem 274(34):24202-24210. https://doi.org/10.1074/ jbc.274.34.24202

30. Ussher JR, Koves TR, Cadete VJ et al (2010) Inhibition of de novo ceramide synthesis reverses diet-induced insulin resistance and enhances whole-body oxygen consumption. Diabetes 59(10): 2453-2464. https://doi.org/10.2337/db09-1293

31. Tan-Chen S, Guitton J, Bourron O, Le Stunff H, Hajduch E (2020) Sphingolipid metabolism and signaling in skeletal muscle: from physiology to physiopathology. Front Endocrinol (Lausanne) 11: 491. https://doi.org/10.3389/fendo.2020.00491

32. Meyer MM, Levin K, Grimmsmann T, Beck-Nielsen H, Klein HH (2002) Insulin signalling in skeletal muscle of subjects with or without type II-diabetes and first degree relatives of patients with the disease. Diabetologia 45(6):813-822. https://doi.org/10.1007/ s00125-002-0830-9

33. Blachnio-Zabielska A, Baranowski M, Zabielski P, Gorski J (2010) Effect of high fat diet enriched with unsaturated and diet rich in saturated fatty acids on sphingolipid metabolism in rat skeletal muscle. J Cell Physiol 225(3):786-791. https://doi.org/10.1002/ jcp. 22283

34. Kien CL, Bunn JY, Poynter ME et al (2013) A lipidomics analysis of the relationship between dietary fatty acid composition and insulin sensitivity in young adults. Diabetes 62(4):1054-1063. https:// doi.org/10.2337/db12-0363

35. Capel F, Cheraiti N, Acquaviva C et al (2016) Oleate dosedependently regulates palmitate metabolism and insulin signaling in C2C12 myotubes. Biochim Biophys Acta 1861(12 Pt A):2000 2010. https://doi.org/10.1016/j.bbalip.2016.10.002

36. Pinel A, Rigaudière JP, Laillet B et al (2016) N-3PUFA differentially modulate palmitate-induced lipotoxicity through alterations of its metabolism in $\mathrm{C} 2 \mathrm{C} 12$ muscle cells. Biochim Biophys Acta 1861(1):12-20. https://doi.org/10.1016/j.bbalip.2015.10.003

37. Chung JO, Koutsari C, Blachnio-Zabielska AU, Hames KC, Jensen MD (2017) Intramyocellular ceramides: subcellular concentrations and fractional De novo synthesis in Postabsorptive humans. Diabetes 66(8):2082-2091. https://doi.org/10.2337/db17-0082

38. Straczkowski M, Kowalska I, Nikolajuk A et al (2004) Relationship between insulin sensitivity and sphingomyelin signaling pathway in human skeletal muscle. Diabetes 53(5):1215-1221. https://doi.org/ 10.2337/diabetes.53.5.1215

39. Serlie MJ, Meijer AJ, Groener JE et al (2007) Short-term manipulation of plasma free fatty acids does not change skeletal muscle concentrations of ceramide and glucosylceramide in lean and overweight subjects. J Clin Endocrinol Metab 92(4):1524-1529. https:// doi.org/10.1210/jc.2006-2347

40. Skovbro M, Baranowski M, Skov-Jensen C et al (2008) Human skeletal muscle ceramide content is not a major factor in muscle insulin sensitivity. Diabetologia 51(7):1253-1260. https://doi.org/ 10.1007/s00125-008-1014-z

41. Chavez JA, Summers SA (2003) Characterizing the effects of saturated fatty acids on insulin signaling and ceramide and diacylglycerol accumulation in 3T3-L1 adipocytes and $\mathrm{C} 2 \mathrm{C} 12$ myotubes. 
Arch Biochem Biophys 419(2):101-109. https://doi.org/10.1016/j. abb.2003.08.020

42. Coen PM, Goodpaster BH (2012) Role of intramyocelluar lipids in human health. Trends Endocrinol Metab 23(8):391-398. https:// doi.org/10.1016/j.tem.2012.05.009

43. Roden M, Perseghin G, Petersen KF et al (1996) The roles of insulin and glucagon in the regulation of hepatic glycogen synthesis and turnover in humans. J Clin Invest 97(3):642-648. https://doi. org/10.1172/jci118460

44. Lambert JE, Parks EJ (2012) Postprandial metabolism of meal triglyceride in humans. Biochim Biophys Acta 1821(5):721-726. https://doi.org/10.1016/j.bbalip.2012.01.006

45. van Herpen NA, Schrauwen-Hinderling VB, Schaart G, Mensink RP, Schrauwen P (2011) Three weeks on a high-fat diet increases intrahepatic lipid accumulation and decreases metabolic flexibility in healthy overweight men. J Clin Endocrinol Metab 96(4):E691E695. https://doi.org/10.1210/jc.2010-2243

46. Brehm A, Krssak M, Schmid AI, Nowotny P, Waldhausl W, Roden M (2006) Increased lipid availability impairs insulin-stimulated ATP synthesis in human skeletal muscle. Diabetes 55(1):136-140

47. Anderson EJ, Lustig ME, Boyle KE et al (2009) Mitochondrial $\mathrm{H} 2 \mathrm{O} 2$ emission and cellular redox state link excess fat intake to insulin resistance in both rodents and humans. J Clin Invest 119(3):573-581. https://doi.org/10.1172/jci37048

48. Grey NJ, Karl I, Kipnis DM (1975) Physiologic mechanisms in the development of starvation ketosis in man. Diabetes 24(1):10-16. https://doi.org/10.2337/diab.24.1.10

Publisher's note Springer Nature remains neutral with regard to jurisdictional claims in published maps and institutional affiliations. 